\title{
Impact of caregiver burden on health-related quality of life and family functioning of carers of children with epilepsy at the Charlotte Maxeke Johannesburg Academic Hospital, South Africa
}

\author{
U A Sabo, ${ }^{1} \mathrm{MB}$ BS, MPH; P Buttner, ${ }^{2} \mathrm{MSc}, \mathrm{PhD}$; G Scher, ${ }^{3} \mathrm{MB}$ BCh, MMed \\ ${ }^{1}$ Department of Paediatrics, Faculty of Clinical Sciences, Bayero University Kano and Aminu Kano Teaching Hospital, Kano, Nigeria \\ ${ }^{2}$ Centre for Chronic Disease Prevention, James Cook University, Cairns, Australia \\ ${ }^{3}$ Department of Paediatrics and Child Health, Faculty of Health Sciences, Charlotte Maxeke Johannesburg Academic Hospital and University of \\ the Witwatersrand, Johannesburg, South Africa
}

Corresponding author: U A Sabo (uasabo.pae@buk.edu.ng)

\begin{abstract}
Background. The impact of caring for a child with a chronic disease on caregivers and their family functioning contributes to the child's adaptation to the disease.

Objectives. To determine the impact of caregiver burden on the health-related quality of life (HRQOL) and family functioning of carers of children with epilepsy (CWE), and to determine factors associated with a high impact of caregiver burden.

Method. A cross-sectional study was conducted among primary caregivers of CWE attending the Charlotte Maxeke Johannesburg Academic Hospital, South Africa. Participants had been involved in childcare for at least 6 months before study enrolment and all gave informed consent. Data regarding sociodemographic and epilepsy-related variables were obtained from questionnaires, including the 36-item family impact module of the Pediatric Quality of Life assessment tool. Scores in the lower quartile were considered indicative of a negative impact on HRQOL and poor family functioning.

Results. Participants identified as experiencing a high impact of paediatric epilepsy care reported raw scores $\leq 31.3$ for both caregiver burden and family functioning. The family functioning score correlated strongly with the caregivers' HRQOL score $(p=0.78 ; p<0.001)$. Multivariate analysis identified a low level of education among caregivers and a high seizure frequency in patients as independent predictors of caregiver burden associated with a negative impact.

Conclusion. Our findings suggest that the burden of caregiving in paediatric epilepsy among our study population impacts negatively on family functioning. The burden of care was associated with a low level of caregiver education and a high seizure frequency in their children.
\end{abstract}

S Afr J Child Health 2020;14(2):66-70. https://doi.org/10.7196/SAJCH.2020.v14i2.1603

More than $80 \%$ of the 50 million people with epilepsy live in lowand middle-income countries. ${ }^{[1]}$ Approximately $50 \%$ of all types of epilepsy have an onset before the age of 5 years and $75 \%$ present by the age of 20 years. ${ }^{[2]}$ Caring for a child affected by a chronic health condition such as epilepsy may impose a high level of stress on caregivers. This can result in considerable dysfunction in the affected family and may adversely affect the child's adaptation to the disease. Camfield et al. ${ }^{[3]}$ reported a negative correlation between paediatric epilepsy and quality of life of both the child and the caregiver. Similarly, in a study to determine the impact of epilepsy on families and factors associated with a high impact of caregiver burden in a developing country such as India, Datta et al. ${ }^{[4]}$ found that $42 \%$ of the participants were adversely affected by caregiving.

Caregivers of children with epilepsy (CWE) may experience chronic fatigue, sleep deprivation, a lack of control over day-to-day events and insufficient time to complete daily tasks owing to the demands of caregiving. The demands of caregiving and child advocacy, together with stigmatisation, have been linked to restricting leisure and social activities among caregivers. Such social restriction, coupled with poor communication with a partner or spouse, may cause strain in a relationship or marriage; a higher divorce rate has been reported among parents of CWE than among the general population. ${ }^{[5]}$ Caregivers of CWE are often concerned about side-effects of medication, possible cognitive deficits, learning difficulties, and the child's future and career outlook. Moreover, the economic burden of paediatric epilepsy depends largely on the individual setting (e.g. access to a functional medical insurance scheme or social security system, or whether care is financed out of pocket).

To our knowledge, studies have not previously assessed the health-related quality of life (HRQOL) and family functioning of caregivers of CWE in South Africa (SA). Periodic assessment of the effect of childhood epilepsy on caregivers can help to improve the understanding of the healthcare needs and coping mechanisms of families of CWE in SA, which, in turn, can contribute to the management of paediatric epilepsy. This study set out to determine the impact of caregiver burden on carers' HRQOL and family functioning and also to identify factors associated with a high impact of caregiver burden.

\section{Methods}

\section{Study design}

We conducted a cross-sectional study at the paediatric epilepsy outpatient clinic of the Charlotte Maxeke Johannesburg Academic Hospital, SA, between October and December 2015. The hospital is a tertiary teaching centre that receives referrals from Johannesburg and neighbouring provinces. 


\section{Operational definitions}

A caregiver was defined as a member of the patient's social network (family, friends or associates) who is at least 18 years old and primarily responsible for everyday care of the patient. ${ }^{[6]}$ Caregiver burden refers to physical, psychological, social or financial difficulties a caregiver may experience. ${ }^{[7]}$ Seizure frequency was categorised as zero (no seizures per month), low (up to four complex partial and one generalised seizure, or up to $20 \mathrm{partial} /$ myoclonic/absence seizures per month) or high (five or more complex partial, more than one generalised seizure or more than $20 \mathrm{partial} /$ myoclonic/absence seizures per month). ${ }^{[8,9]}$ The number of anti-epileptic drugs (AEDs) taken by the child was classified as none (no AED administered), monotherapy (one AED administered) or polytherapy (two or more AEDs administered). Social support was defined as the support available from other individuals, groups and the larger community. ${ }^{[10]}$ As no universal cut-off values for defining high- and low-impact groups are available based on the Pediatric Quality of Life (PedsQL) scale, the 25th and 75th percentiles of the distribution of the current data (corresponding to the lower and upper quartiles, respectively) were used. A negative impact or poor family functioning was defined as the HRQOL, family functioning or total functioning score being in the lower quartile.

\section{Participants}

Caregivers who had been caring for a CWE for at least 6 months and gave informed consent were included in the study. Caregivers of children with comorbidities such as severe to profound intellectual disability, sickle cell disease or congenital heart disease and those who had experienced a major life event in the past 3 months, such as separation, divorce or retrenchment, were excluded.

\section{Data collection}

The Impact of Paediatric Epilepsy Scale (IPES) is a disease-specific measure with good practicality. It measures the child's HRQOL and the caregiver's concern about the child's future. ${ }^{[8]}$ However, it does not assess the HRQOL of the caregiver or the functioning of the family as a unit. In contrast, the family impact module of the PedsQL questionnaire is a generic measure, consisting of 36 items, and designed to assess the effect of chronic medical conditions on caregivers and their families. It was used in this study owing to the lack of an epilepsy-specific caregiver questionnaire and exhibits adequate psychometric properties regarding the reliability, internal consistency and validity of the construct in low- and middleincome countries. ${ }^{[1]}$ The questionnaire consists of eight scales in total:

- Six scales measure a caregiver's self-reported functioning, namely physical functioning (six items), emotional functioning (five items), social functioning (four items), cognitive functioning (five items), communication (three items) and worry (five items)

- Another two scales measure a caregiver's reported family functioning, namely daily activities (three items) and family relationships (five items).

The scales all have five Likert-type response options, namely 'never', 'almost never,' 'sometimes', 'often' and 'almost always'. The response options correspond to scores of 100, 75, 50, 25 and 0, respectively. The total functioning score is calculated as the sum of the 36 items divided by the number of items answered.

The HRQOL summary score was considered a reflection of the impact of epilepsy on the HRQOL of a caregiver and was calculated as the sum of the 20 items on the physical, emotional, social and cognitive functioning scales divided by the number of items answered. The family functioning summary score was considered a reflection of the impact of epilepsy specifically on family activities and relationships and was calculated as the total score from the items related to daily activities and family relationships divided by the number of items answered. Given the interpretation of the scales, higher scores indicated better functioning (less negative impact).

On each clinic day, the first 10 eligible caregivers were recruited. However, on the last clinic day of the year, we could enrol only nine caregivers owing to low turnout. The sociodemographic characteristics of the caregivers (age, gender, religion, education, employment status, housing and sources of social support) were recorded. The patient's clinical information, such as age at onset of epilepsy, duration of epilepsy, average number of seizures per month in the preceding 3 months, number of AEDs administered, electroencephalograms and neuroimaging results, were recorded separately. The questionnaire was administered by trained interviewers (the lead researcher and a retired nurse).

\section{Statistical analysis}

The mean (standard deviation (SD)) score for each of the three scales in the family impact module of the PedsQL questionnaire was calculated for each participant according to the developer's guidelines. $^{[12]}$ Numerical data were reported as means and SDs when symmetrically distributed and as medians and interquartile ranges (IQRs) when skewed. Categorical data were presented as absolute and relative frequencies. Bivariate associations between characteristics were investigated using unpaired $t$-tests, one-way analysis of variance, $\chi^{2}$ tests, Spearman's rank correlation and nonparametric Kruskal-Wallis and Mann-Whitney $U$ tests. The HRQOL and family functioning scores were categorised using the quartiles of their distribution. The new variables were compared using a $\chi^{2}$ test.

Multivariate regression analysis was used to identify independent associations between the characteristics of the caregivers or children and the total functioning score of the caregivers. Categorical variables were dummy coded for this analysis. All characteristics were initially investigated as independent associations. After a model had been identified, the remaining variables were assessed for confounding. Confounding was identified when estimates changed by $5 \%$ or more. The presented model was adjusted for confounding effects.

All statistical analyses assumed $p<0.05$ to indicate statistical significance. Analysis was performed using SPSS (IBM Corp., USA).

\section{Ethical considerations}

Ethical permission for the study was obtained from the Human Research Ethics Committee of the University of the Witwatersrand (ref. no. M150658). Written informed consent was obtained from all participants.

\section{Results}

The mean (SD) age of the participants $(N=109)$ was 37.9 (9.1) years, as shown in Table 1 . The majority of caregivers $(n=68$; $62.4 \%$ ) had completed high school. The median (IQR) duration of epilepsy was $5(2.5-8)$ years. Only $39.4 \%$ of the children $(n=43)$ had had no seizures in the preceding 3 months. The median (IQR) caregiver HRQOL was $46.3(31.3$ - 67.5) and the median (IQR) family functioning score was 46.9 (31.3 - 71.9). The mean (SD) total functioning score of the participants was 48.0 (23.6) (Table 1).

Raw scores $\leq 31.3$ on the HRQOL and family functioning scales were considered to show a high impact of caregiver burden (Table 2). The family functioning score of the participants was strongly correlated with the HRQOL score of the caregivers (Spearman's rank 
Table 3. Multivariate linear regression of factors associated with a high impact of caregiver burden $(N=109)$

\begin{tabular}{|c|c|c|c|c|}
\hline Characteristic & $n$ & $\begin{array}{l}\text { Total functioning } \\
\text { score (mean (SD)) }\end{array}$ & $\begin{array}{l}\text { Standardised } \\
\text { coefficient }\end{array}$ & $p$-value \\
\hline \multicolumn{5}{|l|}{ Level of education of caregiver } \\
\hline Primary school${ }^{\star}$ & 18 & $30.5(18.7)$ & & \\
\hline Junior or high school & 71 & $47.8(22.8)$ & 0.258 & 0.015 \\
\hline Diploma, graduate or higher & 20 & $64.4(19.0)$ & 0.425 & $<0.001$ \\
\hline \multicolumn{5}{|c|}{ Patient's seizure frequency (per month) } \\
\hline Zero $^{*}$ & 43 & $60.8(23.1)$ & & \\
\hline Low $^{\dagger}$ & 33 & $46.6(19.7)$ & -0.263 & 0.003 \\
\hline High $^{\ddagger}$ & 33 & $32.7(18.2)$ & -0.441 & 0.001 \\
\hline \multicolumn{5}{|c|}{$\begin{array}{l}\mathrm{SD}=\text { standard deviation. } \\
{ }^{*} \text { Reference category. } \\
{ }^{+} \text {Up to four complex partial, one generalised or up to } 20 \mathrm{partial} / \mathrm{myoclonic} / \text { absence seizures per month. } \\
{ }^{*} \text { Five or more complex partial, one generalised or }>20 \mathrm{partial} / \mathrm{myoclonic} / \text { absence seizures per month. }\end{array}$} \\
\hline
\end{tabular}

Table 4. Scores of caregivers of children with poorly and well-controlled epilepsy $(N=109)$ for health-related quality of life, family functioning and worry and communication

\begin{tabular}{|c|c|c|c|c|c|c|}
\hline \multirow[b]{2}{*}{ Scale } & \multicolumn{2}{|c|}{ Zero seizure frequency $(n=43)$} & \multicolumn{2}{|c|}{ Low seizure frequency $(n=33)$} & \multicolumn{2}{|c|}{ High seizure frequency $(n=33)$} \\
\hline & Median (IQR) & Mean (SD) & Median (IQR) & Mean (SD) & Median (IQR) & Mean (SD) \\
\hline \multicolumn{7}{|c|}{ HRQOL scores of caregivers } \\
\hline Total score & $60.0(45.0-81.3)$ & $61.2(23.0)$ & $50.0(35.7-65.6)$ & $50.2(21.9)$ & $31.5(24-38.8)$ & $32.8(17.5)$ \\
\hline Physical & $60.0(41.7-80.0)$ & $59.4(22.1)$ & $50.0(35.5-70.0)$ & $50.4(22.4)$ & $30.0(20.0-40.8)$ & $31.7(18.7)$ \\
\hline Emotional & $55.0(40.0-75.0)$ & $56.4(23.1)$ & $40.0(30-60.75)$ & $44.7(20.1)$ & $30.0(22.5-34.2)$ & $29.6(17.9)$ \\
\hline Social & $64.2(50.0-87.0)$ & $64.5(23.7)$ & $50.0(35.8-65.2)$ & $51.2(22.2)$ & $35.0(25.0-41.9)$ & $34.0(18.1)$ \\
\hline Cognitive & $68.8(45.0-88.0)$ & $64.6(25.3)$ & $50.0(37.5-75)$ & $54.5(24.6)$ & $35.0(29.9-42.5)$ & $36.3(17.4)$ \\
\hline Worry & $50.5(36.0-70.0)$ & $51.9(23.1)$ & $35.4(20.0-54.8)$ & $38.5(20.1)$ & $25.0(12.6-35.0)$ & $29.2(24.6)$ \\
\hline Communication & $60.4(40.4-80.0)$ & $60.4(26.3)$ & $41.7(31.3-62.5)$ & $45.2(20.4)$ & $30.0(15.0-41.8)$ & $33.5(24.7)$ \\
\hline \multicolumn{7}{|c|}{ Family functioning scores } \\
\hline Total score & $65.6(42.5-90.6)$ & $65.0(27.0)$ & $46.9(15.6-71.9)$ & $47.9(27.8)$ & $35.0(18.0-48.4)$ & $34.2(18.9)$ \\
\hline Activity & $56.3(33.8-87.5)$ & $60.4(27.6)$ & $37.5(14.8-66.5)$ & $42.6(27.1)$ & $30.5(12.3-38.5)$ & $28.3(16.9)$ \\
\hline Relationship & $70.8(47.5-100)$ & $69.6(27.2)$ & $55.0(20.5-75)$ & $53.5(28.5)$ & $40.0(20.5-55.0)$ & $38.2(20.6)$ \\
\hline
\end{tabular}

demands may further also limit caregivers' ability to pursue their own interests, which may translate to a poor HRQOL. ${ }^{[15]}$ Similarly, parental beliefs and attitudes concerning epilepsy, together with the quality of parent-physician relationships, have been reported to be significantly associated with caregivers' HRQOL. ${ }^{[16]}$

Our data show a strong positive correlation between the caregivers' HRQOL and family functioning scores; two-thirds of caregivers who experienced a negative impact of caregiver burden also reported poor family functioning. This observation may be related to the risk of poor communication, cohesion (a low level of emotional connection with other family members) and poor integration among caregivers who report poor HRQOL. In turn, these factors may be associated with deteriorating family functioning. ${ }^{[17]}$ The findings merit further exploration to determine whether a low caregiver HRQOL score could be used as a predictor of poor family functioning, and vice versa.

Our results show that the higher the education level of the caregiver, the less the impact of paediatric epilepsy on both the carer and their families. Education is a resource that aids in acquisition, processing and organisation skills. ${ }^{[18]}$ A low level of caregiver education has previously been reported among families who reported a negative impact of paediatric epilepsy. ${ }^{[19]}$ Judge ${ }^{[20]}$ similarly found an association between maternal education levels and families' efforts to be active and innovative in managing paediatric disability. Improved caregiver education may also help change parental beliefs, attitudes and the perception of stigma around epilepsy, facilitate access to educational materials and support groups through social media networks, and enhance parent-physician relationships.

High seizure frequency was significantly associated with a negative impact of caregiver burden in the current study. This finding is congruent with those from previous studies ${ }^{[4,8,19,21]}$ and could be due to a number of factors. For example, caregivers of children with frequent seizures may experience limited time to spend with their spouses or friends or to develop personal interests. ${ }^{[22,23]}$ Uncontrolled seizures may also severely affect the carer's situation at work owing to frequent calls from the child's school when the child has a seizure and an increased risk of discrimination and stigma at work and in social settings. ${ }^{[23]}$ Parents have described witnessing their child's seizures as a highly anxiety-provoking experience. Mothers of children with intractable seizures often have a high level of parenting stress and in one study, nearly two-thirds of these mothers have been found to be in the clinical range of total stress on the parenting stress index. ${ }^{[15]}$

Our findings show that caregivers of children with a high seizure frequency reported significantly lower HRQOL scores on all scales compared with those with a low seizure frequency; caregivers of children who experienced no seizures in the preceding 3 months had the highest HRQOL scores. Lv et al. ${ }^{[23]}$ reported similar findings across all scales when comparing the effect of good and poor seizure control. 
In our study, family functioning scores were found to be highest when social support was provided by family and friends, independent of a CSG being available, and scores were lowest when only a CSG was available. Social support is a valuable resource for families caring for a child with a disability. ${ }^{[20]}$ Social support from personal networks (extended family and friends) has been found to have the strongest relationship to any number of outcomes. Judge ${ }^{[20]}$ suggests that there is a considerable positive impact on parent self-efficacy and personal control appraisals when professional support practices mirror the features of informal support. When support from extended family and friends is not available, caregivers may benefit from the formal social support provided by nurses, psychologists and other healthcare providers. ${ }^{[18]}$

Our study was the first to evaluate caregiver burden associated with paediatric epilepsy among SA families. Raw scores provide reference values that may prove useful when this assessment tool is used in similar settings. Using an interviewer method has the advantage of maximising response rates, resulting in few, if any, missing items, and minimising errors of understanding. Multivariate analysis allowed us to identify predictors of high impact from the associated factors.

\section{Study limitations}

We had to use cut-off values without knowledge of comparative values for carers of healthy controls. The PedsQL assessment is a validated scale and is widely used. However, cut-offs to define groups who experience high or low impacts have not been generally described. According to the theory of psychometric testing, the actual values of scales depend on the population in which they are applied and hence cut-off values cannot easily be transferred from one population to another. ${ }^{[24]}$ Previous analyses using the PedsQL instrument reported cut-off values as mean$1 \mathrm{SD}^{[25]}$ and mean-2 $\mathrm{SDs}^{[14]}$ compared with healthy controls to define negative impact. These values would not translate to the data from our study as the mean values were considerably higher (30 units) in these (US) studies. Moreover, the distribution of the PedsQL scores in our study was skewed and hence the results were described using medians and IQRs rather than means and SDs. Thus, the 25th and 75th percentiles were used to define impact categories without the knowledge of scores associated with caretakers of healthy children.

\section{Conclusion}

Our findings suggest that paediatric epilepsy is associated with a negative impact of caregiver burden, especially among carers with low education levels and carers of children with a high seizure frequency. Healthcare professionals and support groups should be encouraged to provide social support to families caring for CWE to help alleviate the demands of constant caregiving.

\section{Declaration. None.}

Acknowledgements. We thank the MAPI Trust for making available the family impact module of the PedsQL questionnaire. We also acknowledge training by Dorothy L. Southern on scientific writing and for critically reviewing this manuscript.

Author contributions. UAS conceptualised the study and contributed to data collection and analysis and manuscript preparation. PB led the statistical analysis, wrote the result section of the manuscript and was responsible for critical review of the manuscript. GS critically reviewed the concept of the study and the manuscript prior to submission.
Funding. None.

Conflicts of interest. None.

1. World Health Organization. Epilepsy: Aetiology, epidemiology and prognosis. https://www.who.int/news-room/fact-sheets/detail/epilepsy (accessed 18 September 2017)

2. Olubunmi AO. Epilepsy in Nigeria - a review of etiology, epidemiology and management. BJPM 2006;8:27-51. https://doi.org/10.4314/bjpm.v8i1.47362

3. Camfield C, Breau L, Camfield P. Assessing the impact of pediatric epilepsy and concomitant behavioral, cognitive, and physical/neurologic disability: Impact of Childhood Neurologic Disability Scale. Dev Med Child Neurol 2003;45(3):152-159. https://doi.org/10.1111/j.1469-8749.2003.tb00923.x

4. Datta SS, Premkumar TS, Fielding S, et al. Impact of pediatric epilepsy on Indian families: Influence of psychopathology and seizure related variables. Epilepsy Behav 2006;9(1):145-151. https://doi.org/10.1016/j.yebeh.2006.04.011

5. Lach LM, Ronen GM, Rosenbaum PL, et al. Health-related quality of life in youth with epilepsy: Theoretical model for clinicians and researchers. Part I: The role of epilepsy and co-morbidity. Qual Life Res 2006;15(7):1161-1171. https://doi.org/10.1007/s11136-006-0051-7

6. Karakis I, Cole AJ, Montouris GD, San Luciano M, Meador KJ, Piperidou C. Caregiver burden in epilepsy: Determinants and impact. Epilepsy Res Treat 2014;2014:808421. https://doi.org/10.1155/2014/808421

7. George LK, Gwyther LP. Caregiver well-being: A multidimensional examination of family caregivers of demented adults. Gerontologist 1986;26(3):253-259. https://doi.org/10.1093/geront/26.3.253

8. Camfield C, Breau L, Camfield P. Impact of pediatric epilepsy on the family: A new scale for clinical and research use. Epilepsia 2001;42(1):104-112. https:// doi.org/10.1046/j.1528-1157.2001.081420.x

9. Baker GA, Camfield C, Camfield P, et al. Commission on Outcome Measurement in Epilepsy, 1994 - 1997: Final report. Epilepsia 1998;39(2):213231. https://doi.org/10.1111/j.1528-1157.1998.tb01361.x

10. Ozbay F, Johnson DC, Dimoulas E, Morgan CA, Charney D, Southwick S. Social support and resilience to stress: From neurobiology to clinical practice. Psychiatry (Edgmont) 2007;4(5):35-40.

11. Scarpelli AC, Paiva SM, Pordeus IA, Varni JW, Viegas CM, Allison PJ. The pediatric quality of life inventory (PedsQL) family impact module: Reliability and validity of the Brazilian version. Health Qual Life Outcomes 2008;6(1):35. https://doi.org/10.1186/1477-7525-6-35

12. Varni JW. The PedsQL TM Scoring Algorithm; version 14. Lyon, MAPI Trust Research; 2014. http://www.pedsql.org/score.html (accessed 2 April 2015).

13. Isa SI, Aziz AA, Rahman AA, et al. The impact of children with disabilities on parent health-related quality of life and family functioning in Kelantan and its associated factors. J Dev Behav Pediatr 2013;34(4):262-268. https://doi. org/10.1097/dbp.0b013e318287cdfe

14. Varni JW, Bendo CB, Shulman RJ, et al. Interpretability of the PedsQL Gastrointestinal Symptoms Scales and Gastrointestinal Worry Scales in pediatric patients with functional and organic gastrointestinal diseases. J Pediatr Psychol 2015;40(6):591-601. https://doi.org/10.1093/jpepsy/jsv005

15. Wirrell EC, Wood L, Hamiwka LD, Sherman EM. Parenting stress in mothers of children with intractable epilepsy. Epilepsy Behav 2008;13(1):169-173. https://doi.org/10.1016/j.yebeh.2008.02.011

16. Ryan BL, Speechley KN, Levin SD, Stewart M. Parents' and physicians' perceptions of childhood epilepsy. Seizure 2003;12(6):359-368. https://doi. org/10.1016/s1059-1311(02)00325-4

17. Buelow JM, McNelis A, Shore CP, Austin JK. Stressors of parents of children with epilepsy and intellectual disability. J Neurosci Nurs 2006;38(3):147-154 https://doi.org/10.1097/01376517-200606000-00003

18. Shore CP, Austin JK, Dunn DW. Maternal adaptation to a child's epilepsy. Epilepsy Behav 2004;5(4):557-568. https://doi.org/10.1016/j.yebeh.2004.04.015

19. Lagunju IA, Orimadegun AE, Akimbami FO, Brown BJ, Olorundare EI, Ohaeri JU. Health-related quality of life of Nigerian children with epilepsy. Afr J Neurol Sci 2009;28:28-37.

20. Judge SL. Parental coping strategies and strengths in families of young children with disabilities. Family Relations 1998;47(3):263-268. https://doi. org/10.2307/584976

21. Aronu AE, Ojinnaka NC. Psychiatric morbidity among parents of children with epilepsy in Enugu, Nigeria. Neurol Asia 2009;14:15-20.

22. Modi AC. The impact of a new pediatric epilepsy diagnosis on parents: Parenting stress and activity patterns. Epilepsy Behav 2009;14(1):237-242. https://doi.org/10.1016/j.yebeh.2008.10.009

23. Lv R, Wu L, Jin L, et al. Depression, anxiety and quality of life in parents of children with epilepsy. Acta Neurol Scand 2009;120(5):335-341. https://doi. org/10.1111/j.1600-0404.2009.01184.x

24. Streiner DL, Norman GR, Cairney J. Health Measurement Scales. A practical guide to their development and use. Oxford: Oxford University Press, 2014.

25. Varni JW, Burwinkle TM, Seid M, Skaar D. The PedsQL $L^{\mathrm{rm}} 4.0$ as a measure of pediatric population health measure: Feasibility, reliability and validity. Ambul Pediatr 2003;3:329-341. https://doi.org/10.1367/1539-4409(2003)003<0329:tp aapp $>2.0$. co; 2

Accepted 2 September 2019 